\title{
Crescimento inicial de três espécies arbóreas da Floresta Atlântica em resposta à variação na quantidade de $l u z^{1}$
}

\author{
SÔNIA REGINA DUZ², ALEXANDRE SIMINSKI ${ }^{2}$, MARISA SANTOS $^{2} \mathrm{e}$ \\ MARIA TEREZINHA S. PAULILO ${ }^{2,3}$
}

(recebido: 30 de maio de 2001; aceito: 17 de junho de 2004)

\begin{abstract}
Growth response of three woody species seedlings from the Tropical Atlantic rain forest to changing light conditions). The growth response of seedlings of three woody species from the Tropical Atlantic rain forest to changing light conditions was studied. The species were the early successional Cecropia glazioui Sneth., the intermediary in succession Cedrela fissilis Vell. and the late successional Bathysa australis (A. St.-Hil.) Hook. ex Sch. These species showed, within the range of light gradient, plasticity to increase light interception at lower light levels (through increasing leaf area ratio - LAR, and decreasing root/shoot ratio - R/SH); and plasticity to increase carbon gain and decreasing transpiration at high light levels (through increasing stomatal density and R/SH, and decreasing LAR). Species responses to irradiance varied with position along the light gradient. Plants at the lower end of the light gradient were more responsive than plants at the higher end of the light gradient. $C$. glazioui seemed to be more plastic than $C$. fissilis to alter several features. The behavior showed by the three species in response to changing light was consistent with their environmental occurrence.
\end{abstract}

Key words - Atlantic rainforest, growth, light, seedlings, tropical trees

RESUMO - (Crescimento inicial de três espécies arbóreas da Floresta Atlântica em resposta à variação na quantidade de luz). Verificou-se a resposta de crescimento à variação na intensidade de luz de plântulas de três espécies arbóreas da Floresta Tropical Atlântica, Cecropia glazioui Sneth., Cedrela fissilis Vell. e Bathysa australis (A. St.-Hil.) Hook. ex Sch., respectivamente de estádios inicial, intermediário e final de sucessão. As três espécies mostraram, dentro de um determinado gradiente de luz, plasticidade para aumentar a captação de luz quando em baixa irradiância (através de aumento da razão de área foliar -RAF e diminuição da razão entre raiz e parte aérea - R/PA) e plasticidade para aumentar o ganho de carbono e diminuir a transpiração quando em alta irradiância (através dos aumentos da razão R/PAe densidade estomática, e da diminuição da RAF). A plasticidade das espécies em variar determinado parâmetro em função da intensidade de luz foi dependente do gradiente de intensidade de luz aplicado. A plasticidade foi maior nas intensidades mais baixas de luz tanto para C. glazioui quanto para C. fissilis. Para a maior parte dos parâmetros analisados, C. glazioui mostrou maior parte plasticidade para aclimatar-se à maior irradiância, que C. fissilis. As variações apresentadas pelas espécies na morfologia e fisiologia em relação à variação na intensidade de luz são consistentes com o local de ocorrência de cada espécie.

Palavras-chave - arbóreas tropicais, crescimento, Floresta Atlântica, luz, plântulas

\section{Introdução}

A luz é um dos fatores físicos mais importantes no controle do desenvolvimento de plântulas de espécies arbóreas em florestas tropicais úmidas (Lee et al. 1997). As condições de luz para as plântulas, no nível do chão da floresta, são extremamente variáveis. A radiação incidente nas folhas próximas ao chão, devido à atenuação da radiação através dos vários estratos da cobertura vegetal, pode ser cerca de $1 \%$ a $2 \%$ da radiação incidente nas folhas do dossel. Em clareiras,

\footnotetext{
1. Parte da dissertação de mestrado de S.R. Duz

2. Universidade Federal de Santa Catarina, Departamento de Botânica, Caixa Postal 476, 88040-900 Florianópolis, SC, Brasil.

3. Autor para correspondência: paulilo@ccb.ufsc.br
}

provocadas pela queda ou retirada de árvores, a quantidade de luz pode ser similar à incidente no dossel (Lee et al. 1996) e variar da borda para o centro desta, ou mesmo variar com o decorrer do tempo, devido ao crescimento de plantas adjacentes (Kitajima 1996).

Quando as plântulas experimentam uma mudança nas condições de luz, a maioria delas é capaz, em maior ou menor grau, de aclimatar-se à mudança ocorrida (Kitajima 1996). A aclimatação de plantas à quantidade de luz incidente ocorre no sentido de maximizar o ganho total de carbono que pode se dar através de dois caminhos: a) mudança nas propriedades de assimilação de carbono pelas folhas, envolvendo ajustes fisiológicos e morfológicos, e b) mudança no padrão de alocação de biomassa em favor da parte vegetativa mais severamente afetada pela mudança (Osunkoya et al. 1994). Algumas das principais mudanças, decorrentes de aumento na quantidade de luz, são aumento na 
espessura foliar, resultando no aumento da relação massa foliar/área foliar (Lee et al. 1996); aumento na densidade estomática (Lee et al. 2000); maior alocação de biomassa para as raízes (Osunkoya et al. 1994); variação na altura do caule (Poorter 1999) e orientação das folhas (Ishida et al. 1999). A capacidade de aclimatação a mudanças na intensidade de luz é variável de espécie para espécie e pode depender do gradiente de luz que as espécies recebem (Poorter 1999) ou de seu estádio sucessional (Strauss-Debenedetti \& Bazzaz 1994). Há uma infinidade de terminologias para a classificação de espécies em classes sucessionais (Matthes \& Martins 1996), mas, tendo por base a resposta de crescimento das espécies vegetais à luz, costuma-se distinguir dois grupos sucessionais extremos: a) as de estádio inicial de sucessão (pioneiras), que germinam, sobrevivem e crescem somente em clareiras e b) as de estádio final e sucessão (clímax), que germinam e sobrevivem em ambientes sombreados do subosque (Whitmore 1990). Entretanto, entre estes dois extremos, já se reconhece um grande número de espécies, ocupando estádios intermediários na sucessão (Osunkoya et al. 1994).

O grau de plasticidade em relação à variação de luz inerente a cada espécie, pode ter papel fundamental na sobrevivência de plantas em ambientes heterogêneos e variáveis, como o das florestas tropicais, e pode explicar diferenças na distribuição ecológica e geográfica das espécies (Petit et al. 1996). Em vista disso, o presente trabalho analisou respostas morfológicas e fisiológicas, em relação à variação na intensidade de luz, de plântulas de três espécies em diferentes estádios sucessionais, com o intuito de ajudar a explicar a ocorrência e distribuição dessas espécies na natureza. $\mathrm{O}$ trabalho procurou responder às seguintes questões: a) as respostas morfológicas e fisiológicas apresentadas pelas três espécies diferem entre si? b) em que variação de gradiente de luz houve maior plasticidade nas respostas à variação de luz? c) as respostas dadas pelas espécies em relação à variação de luz são coerentes com seu ambiente de ocorrência?

As espécies analisadas foram três arbóreas de diferentes estádios sucessionais da Floresta Ombrófila Densa, Cecropia glazioui Sneth., Cedrela fissilis Vell. e Bathysa australis (A. St.-Hil.) Hook. ex Sch. Cecropia glazioui (embaúba) é espécie encontrada desde a Bahia até o Rio Grande do Sul, ocorrendo na Floresta Ombrófila Densa (Lorenzi 1998). É espécie pioneira, colonizando grandes clareiras e/ou áreas de cultivo abandonadas (Tabarelli et al. 1993). Cedrela fissilis (cedro) é espécie encontrada desde Minas Gerais até o Rio Grande do Sul, principalmente distribuída nas Florestas Estacional Decidual e Ombrófila Densa (Klein 1984). É freqüentemente encontrada em clareiras ou bordas de mata (Durigan et al. 1997) ou em matas secundárias (Klein 1984). Bathysa australis (macuqueiro) é espécie arbórea encontrada deste o Rio de Janeiro até o Rio Grande do Sul, exclusiva da Floresta Ombrófila Densa da Encosta Atlântica do sul do Brasil (Lorenzi 1998). É espécie de subosque (Tabarelli et al. 1993).

\section{Material e métodos}

Material vegetal - Foram utilizadas plantas jovens de Cecropia glazioui Sneth. (Cecropiaceae), Cedrela fissilis Vell. (Meliaceae) e Bathysa australis (A. St.-Hil.) Hook. ex Sch. (Rubiaceae), provindas da germinação de sementes. As sementes de C. glazioui foram coletadas em abril de 1999, no município de Florianópolis (27³5'48’'S e 48³2' 57’'W ), as de B. australis, em maio de 1999, nos municípios de Santo Amaro da Imperatriz (27\%4'17'S e 4846'43'W) e Águas Mornas $\left(27^{\circ} 4^{\prime} 38^{\prime \prime} \mathrm{S}\right.$ e $\left.48^{\circ} 49^{\prime} 25^{\prime \prime} \mathrm{W}\right), \mathrm{SC}$ e as de C. fissilis foram adquiridas, em outubro de 1999, através do Instituto de Pesquisas Florestais (IPEF) - São Paulo. A identificação das espécies $C$. glazioui e $B$. australis foi feita por especialistas do Departamento de Botânica da Universidade Federal de Santa Catarina (UFSC), através de material vegetal retirado de árvores cujas sementes foram coletadas para o trabalho. Obtenção de plântulas e condições de crescimento - As sementes das três espécies, mantidas em vidros fechados a $5{ }^{\circ} \mathrm{C}$ até serem utilizadas nos experimentos, foram desinfetadas com hipoclorito de sódio a $5 \%$ por 5 minutos, lavadas em água corrente e colocadas para germinar em bandejas contendo papel de filtro umedecido com solução do fungicida Benlate, na concentração de $100 \mathrm{mg} . \mathrm{L}^{-1}$. As sementes de C. glazioui e as de B. australis foram colocadas para germinar em início de setembro e as de $C$. fissilis, em início de novembro de 1999. As bandejas foram recobertas com plástico transparente e mantidas em laboratório sob luz fluorescente branca, com densidade de fluxo de fótons, entre 400 e $700 \mathrm{~nm}$, de $3 \mu \mathrm{mol}$ de fótons $\mathrm{m}^{-2} \mathrm{~s}^{-1}$ e fotoperíodo de oito horas, a $24^{\circ} \mathrm{C}$ \pm 2 . A luz é necessária para a germinação de sementes de C. glazioui (Valio \& Scarpa 2001). Sementes de C. fissilis são indiferentes à luz para germinar (Lorenzi 1998). No caso de B. australis, um teste preliminar com sementes colocadas para germinar nas mesmas condições descritas acima, só que em bandejas recobertas com papel alumínio (quatro repetições de 50 sementes), mostrou que as sementes não germinam no escuro. Após a germinação das sementes (sete dias para C. glazioui e $C$. fissilis e 30 dias para B. australis) as plântulas obtidas foram transplantadas para bandejas perfuradas contendo solo florestal e vermiculita na proporção de $2: 1$ e mantidas em laboratório nas mesmas condições de luz e temperatura acima descritas por um período de 80 dias 
(C. glazioui), 20 dias (C. fissilis) e 215 dias (B. australis) para seu estabelecimento. As plântulas foram irrigadas diariamente. Após o período de estabelecimento, as plântulas foram uniformizadas por altura de parte aérea, para diminuir a variação intraespecífica e nove delas foram separadas para comporem a primeira coleta. $\mathrm{O}$ restante das plantas uniformes foram colocadas em sacos plásticos pretos, de $20 \times 7 \mathrm{~cm}$, contendo solo florestal e compostagem termofílica na proporção de 1:1. Os sacos plásticos, contendo uma planta cada foram colocados sobre quatro bancadas de madeira de $1,10 \mathrm{~m}^{2}$, a $30 \mathrm{~cm}$ de altura do solo. Sobre cada bancada, contendo 100 plantas por $\mathrm{m}^{2}$, foi colocada uma caixa de $1 \mathrm{~m}^{3}$, confeccionada com tela sombrite que permitia a passagem de $50 \%, 30 \%, 15 \%$ ou $2 \%$ da luz solar incidente, dependendo do tratamento de luz aplicado. As bancadas com as caixas de sombrite foram colocadas a céu aberto, de maneira a evitar o auto-sombreamento, em área do Departamento de Botânica - UFSC/Florianópolis. As plantas de C. glazioui e C. fissilis permaneceram nestas condições de dezembro de 1999 a abril de 2000 e as de $B$. australis de abril a agosto de 2000 , sendo irrigadas diariamente. As plantas foram mudadas de posição dentro das caixas para evitar o sombreamento de umas sobre as outras. Atemperatura máxima nas caixas variou entre $32,5^{\circ} \mathrm{C}$ e $33{ }^{\circ} \mathrm{C}$ e a mínima, entre $18,5^{\circ} \mathrm{C}$ e $21^{\circ} \mathrm{C}$, sendo sempre as maiores temperaturas em caixas com menor sombreamento de luz. As medidas de atenuação de luz foram determinadas através de um quantômetro LICOR 250, com sensor para a faixa de radiação entre 400 e $700 \mathrm{~nm}$, tomando-se a densidade de fluxo de fótons de radiação fotossinteticamente ativa (RFA) a pleno sol e sob as telas sombrite, calculando-se, então, a porcentagem de luz transmitida. A densidade de fluxo de fótons a pleno sol e em cada nível de luz foi medida ao meio dia, de um dia claro, próximo ao solstício de verão e em dia típico de outono, sendo o valor máximo a pleno sol, no primeiro caso, $1.800 \mu \mathrm{mol}$ de fótons $\mathrm{m}^{-2} \mathrm{~s}^{-1} \mathrm{e}$, no segundo caso, $1.200 \mu \mathrm{mol}$ de fótons $\mathrm{m}^{-2} \mathrm{~s}^{-1}$. A proporção de vermelho e vermelho extremo em cada nível de luz foi medida com quantômetro (SKYE Instruments) com sensor para radiação de $660 \mathrm{~nm}$ e $730 \mathrm{~nm}$ (sensor SKR 110).

Número de coletas - Para C. glazioui e C. fissilis foram realizadas três coletas, a primeira em $1^{\circ}$ de dezembro, imediatamente antes de as plântulas serem transplantadas das condições de laboratório para os diferentes tratamentos de luz, a segunda, em 17 de fevereiro, após 78 dias em cada nível de luz, e a terceira, em 10 de abril, após 131 dias (C. fissilis), ou 24 de abril, após 146 dias (C. glazioui) em cada nível de luz. Para $B$. australis, foram realizadas duas coletas, a primeira em 28 de abril, imediatamente antes de as plântulas serem transplantadas das condições de laboratório para os diferentes tratamentos de luz e a segunda, em 3 de agosto, aos 93 dias de crescimento em cada nível de luz.

Análise dos dados - $\mathrm{O}$ efeito dos tratamentos de luz sobre C. glazioui e C. fissilis foi analisado através de regressão, enquanto que para B. australis e para os dados de inclinação da folha em $C$. glazioui, o efeito dos tratamentos de luz foi analisado através do teste t-Student $(\mathrm{p}<0,05)$, segundo Centeno (1982). No caso de B. australis só foi possível obter número suficiente de plantas uniformes para os tratamentos de $30 \%$ e $2 \%$ de luz. Foram utilizadas para as medidas nove plantas por tratamento $(\mathrm{n}=36)$, a não ser quando especificado de maneira diferente. São mostrados no trabalho apenas os valores para a última coleta.

Medidas de massa seca, área foliar e comprimento do caule Para a obtenção da massa seca, as plantas, separadas em raiz, caule, pecíolo e lâmina foliar, foram colocadas em estufa a $80^{\circ} \mathrm{C}$ por 48 horas, sendo, após este período, as diferentes partes vegetais pesadas separadamente. A área foliar foi obtida escaneando-se as lâminas foliares das plantas e calculando-se a área da imagem obtida com o auxílio do programa computacional IDRISI para medidas de área (Eastman 1997). O comprimento do caule (altura), foi determinado com uma régua milimetrada, medindo-se a distância entre a transição raiz/caule e a base da gema apical. Análise de crescimento - As taxas médias de crescimento relativo (TCR), de assimilação líquida (TAL) e a razão média de área foliar (RAF) foram calculadas entre a primeira e a última coletas. A TCR calculada pela equação (ln $M_{2}$ $\left.\ln \mathrm{M}_{1}\right) /\left(\mathrm{T}_{2}-\mathrm{T}_{1}\right)$, a TAL, pela equação $\left[\left(\mathrm{M}_{2}-\mathrm{M}_{1}\right) /\left(\mathrm{T}_{2}-\mathrm{T}_{1}\right)\right] \times$ $\left[\left(\ln A_{2}-\ln A_{1}\right) /\left(A_{2}-A_{1}\right)\right]$, a RAF, pela equação $\left[\left(A_{1} / M_{1}\right)+\right.$ $\left.\left(\mathrm{A}_{2} / \mathrm{M}_{2}\right)\right] / 2$, a massa foliar específica, pela equação $\mathrm{MFE}=\mathrm{M}_{\text {foliar }} / \mathrm{A}$ e a razão de massa foliar, pela equação RMF $=\mathrm{M}_{\text {foliar }} / \mathrm{M}_{\text {total }}$ (Hunt 1982). $\mathrm{M}$ representa a massa seca, $\mathrm{T}$ representa o tempo $(1=$ primeira coleta e 2 = última coleta $)$ e A representa a área foliar. MFE e RMF foram calculadas apenas para a última coleta.

Determinação da densidade e tamanho de estômatos - Para plantas de $C$. fissilis e de $B$. australis, réplicas da epiderme abaxial da folha foram retiradas com esmalte incolor para unhas e montadas em lâmina e lamínula. Em $C$. glazioui, devido à grande quantidade de tricomas na face abaxial, porções de folhas foram clarificadas através de fervura em álcool a $96^{\circ} \mathrm{GL}$, lavadas em água destilada e colocadas em solução de Jeffrey por três horas para dissociação dos tecidos (Johansen 1940). Pequenos pedaços de epiderme da face abaxial foram coletados com o auxílio de um pincel e corados com Giemsa, sendo então montados em lâmina e lamínula com glicerina liquida a $50 \%$. A contagem dos estômatos por $\mathrm{mm}^{2}$ na face abaxial da epiderme (única face com estômatos nas três espécies) foi feita em áreas intercostais da região mediana da folha mais jovem totalmente expandida. As imagens das epidermes obtidas ao microscópio óptico com magnitude de $400 \times$ foram projetadas, com auxílio de câmara-clara acoplada ao microscópio, sobre área demarcada de $200 \times 200 \mu \mathrm{m}$. Foram utilizadas três plantas por nível de luz e uma folha por planta. Para cada folha foram feitas oito repetições, totalizando 24 repetições por nível de luz. Para estes dados foram utilizadas plantas da última coleta.

Determinação do ângulo de inclinação de folhas de C. glazioui - Foram medidos, através de transferidor, os ângulos formados entre a superfície inferior do pecíolo e o 
caule e entre a face inferior da lâmina foliar e o pecíolo. Foram utilizadas seis plantas, crescidas por 146 dias, por intensidade de luz ( $2 \%$ e $50 \%)$.

\section{Resultados}

Razão raiz/parte aérea e altura do caule - A significância da equação e coeficiente de regressão para R/PA versus intensidade de luz (figura 1), mostra que o comportamento da R/PA em relação aos níveis de luz em $C$. glazioui e $C$. fissilis foi o de aumentar com o aumento do nível de luz. B. australis também aumentou significativamente a R/PA com o aumento da intensidade de luz $(0,10 \pm 0,05$ a $2 \%$ de luz e $0,18 \pm 0,10$ a $30 \%$ de luz). A altura do caule (figura 2), em C. glazioui, diminuiu à medida que se aumentou o nível de luz e em C. fissilis sofreu um aumento até o nível de $15 \%$ de luz, decrescendo, a partir daí com o aumento da intensidade de luz. Em B. australis, o comprimento do caule não apresentou diferença significativa com a variação do nível de luz.

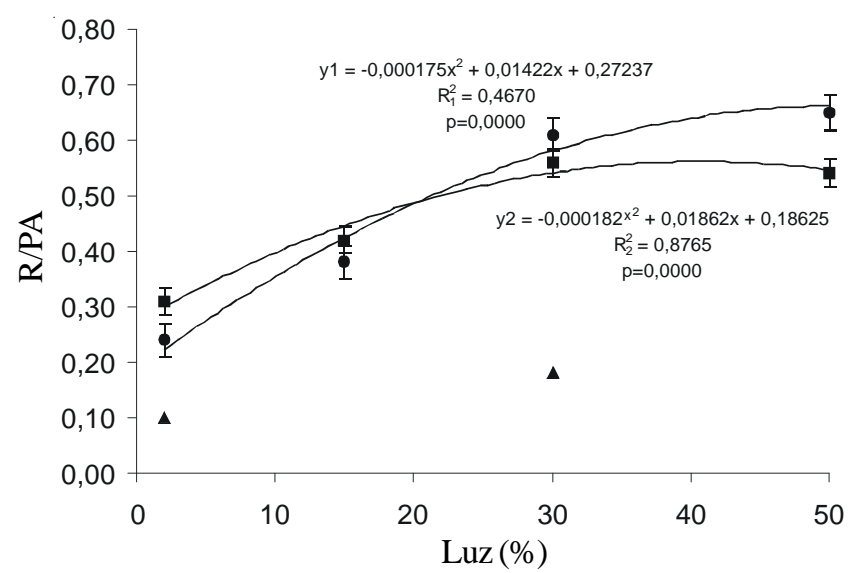

Figura 1. Razão raiz/parte aérea (R/PA) de plantas de Cecropia glazioui $(\bullet)$, Cedrela fissilis $(\bullet)$ e Bathysa australis $(\boldsymbol{\bullet})$ crescidas, respectivamente por 146, 131 e 93 dias em diferentes níveis de luz. Equações de regressão para Cedrela fissilis $\left(\mathrm{y}_{1}\right)$ e para Cecropia glazioui $\left(\mathrm{y}_{2}\right)$, coeficientes de determinação para Cedrela fissilis $\left(\mathrm{R}^{2}{ }_{1}\right)$ e para Cecropia glazioui $\left(\mathrm{R}_{2}{ }_{2}\right)$. Modelos e coeficientes significativos em nível de $5 \%$ de probabilidade. Os índices de dispersão correspondem ao erro padrão da média.

Figure 1. Root/shoot ratio (R/PA) of Cecropia glazioui $(\bullet)$, Cedrela fissilis (-) and Bathysa australis ( $\bullet$ ) seedlings growing respectively during 146, 131 and 93-day period under different light conditions. Regression equations for Cedrela fissilis $\left(\mathrm{y}_{1}\right)$ and for Cecropia glazioui $\left(\mathrm{y}_{2}\right)$, regression coefficients for Cedrela fissilis $\left(\mathrm{R}^{2}{ }_{1}\right)$ and for Cecropia glazioui $\left(\mathrm{R}_{2}{ }_{2}\right)$. Regression curves and regression coefficients are significant at the 0,005 level.
Análise de crescimento - Observa-se pela figura 3 que a curva para a TCR, tanto para $C$. glazioui como para C. fissilis, mostrou aumento do valor da TCR até $30 \%$ de luz, decaindo este valor com o subseqüente aumento da intensidade de luz. Em B. australis, a TCR foi significativamente menor a $2 \%$ de luz $(0,022 \pm$ $\left.0,003 \mathrm{mg} \mathrm{dia}^{-1}\right)$ que a $30 \%$ de luz $\left(0,036 \pm 0,005 \mathrm{mg} \mathrm{dia}^{-1}\right)$ A figura 4 mostra que a TAL para $B$. australis foi significativamente menor a $2 \%\left(0,05 \pm 0,01 \mathrm{mg} \mathrm{cm}^{-2} \mathrm{~d}^{-1}\right)$ que a $30 \%$ de luz $\left(0,12 \pm 0,05 \mathrm{mg} \mathrm{cm}^{-2} \mathrm{~d}^{-1}\right)$. Para C. glazioui a curva mostrou uma ascensão da TAL com o aumento da intensidade de luz, enquanto que para C. fissilis, houve um aumento da TAL com aumento da intensidade de luz até $30 \%$ de luz, decaindo com o subseqüente aumento na intensidade de luz. As curvas representando o comportamento da RAF (figura 5) em relação ao aumento da intensidade de luz mostram, em C. glazioui e C. fissilis, um decréscimo da RAF com aumento até $30 \%$ de luz, estabilizando-se o valor deste parâmetro com subseqüentes aumentos da intensidade de luz. A inclinação da curva representando a variação

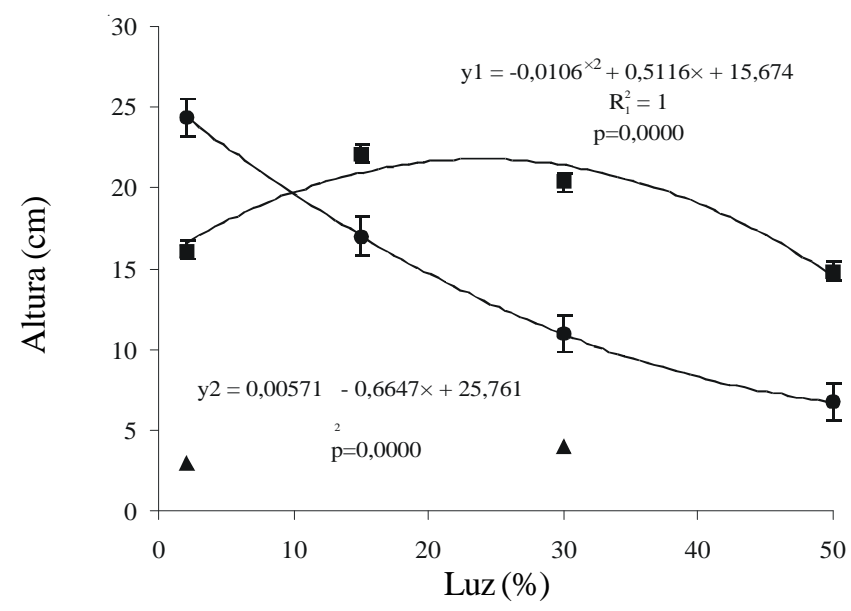

Figura 2. Comprimento do caule de plantas de Cecropia glazioui $(\bullet)$, Cedrela fissilis $(\bullet)$ e Bathysa australis (\) crescidas, respectivamente por 146, 131 e 93 dias em diferentes níveis de luz. Equações de regressão para Cedrela fissilis $\left(\mathrm{y}_{1}\right)$ e para Cecropia glazioui $\left(\mathrm{y}_{2}\right)$, coeficientes de determinação para Cedrela fissilis $\left(\mathrm{R}^{2}{ }_{1}\right)$ e para Cecropia glazioui $\left(\mathrm{R}_{2}{ }_{2}\right)$. Modelos e coeficientes significativos em nível de $5 \%$ de probabilidade. Os índices de dispersão correspondem ao erro padrão da média.

Figure 2. Stem length of Cecropia glazioui (•), Cedrela fissilis (-) and Bathysa australis ( $\mathbf{\Delta})$ seedlings growing respectively during 146, 131 and 93-day period under different light conditions. Regression equations for Cedrela fissilis $\left(\mathrm{y}_{1}\right)$ and for Cecropia glazioui $\left(\mathrm{y}_{2}\right)$, regression coefficients for Cedrela fissilis $\left(\mathrm{R}_{1}^{2}\right)$ and for Cecropia glazioui $\left(\mathrm{R}_{2}^{2}\right)$. Regression curves and regression coefficients are significant at the 0,005 level. 


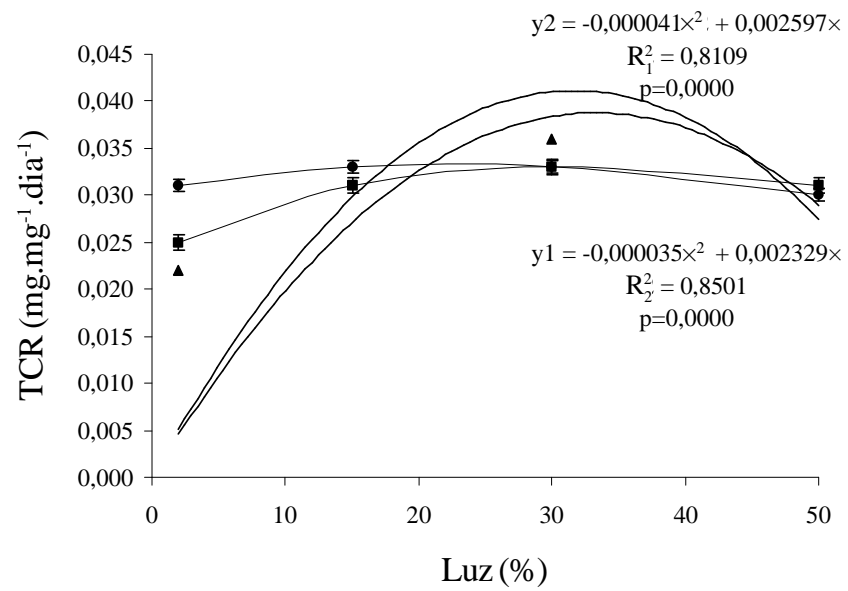

Figura 3. Taxa média de crescimento relativo (TCR) de plantas de Cecropia glazioui (•), Cedrela fissilis (•) e Bathysa australis (ム) crescidas, respectivamente por 146, 131 e 93 dias em diferentes níveis de luz. Equações de regressão para Cedrela fissilis $\left(\mathrm{y}_{1}\right)$ e para Cecropia glazioui $\left(\mathrm{y}_{2}\right)$, coeficientes de determinação para Cedrela fissilis $\left(\mathrm{R}^{2}{ }_{1}\right)$ e para Cecropia glazioui $\left(\mathrm{R}_{2}^{2}\right)$. Modelos e coeficientes significativos em nível de $5 \%$ de probabilidade. Os índices de dispersão correspondem ao erro padrão da média.

Figure 3. Mean relative growth rate (TCR) of Cecropia

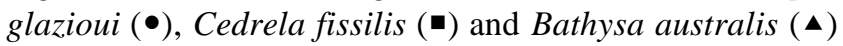
seedlings growing respectively during 146, 131 and 93-day period under different light conditions. Regression equations for Cedrela fissilis $\left(\mathrm{y}_{1}\right)$ and for Cecropia glazioui $\left(\mathrm{y}_{2}\right)$, regression coefficients for Cedrela fissilis $\left(\mathrm{R}_{1}{ }_{1}\right)$ and for Cecropia glazioui $\left(\mathrm{R}_{2}^{2}\right)$. Regression curves and regression coefficients are significant at the 0,005 level.

da RAF com o aumento da intensidade de luz mostrou que $C$. glazioui foi mais sensível à variação da RAF com o aumento da intensidade de luz que $C$. fissilis. B. australis mostrou significativa diminuição da RAF com aumento da intensidade de luz de $2 \%(0,38 \pm$ $\left.0,06 \mathrm{~cm}^{2} \mathrm{mg}^{-1}\right)$ para $30 \%$ de luz $\left(0,27 \pm 0,07 \mathrm{~cm}^{2} \mathrm{mg}^{-1}\right)$. A RMF (figura 6) tende a diminuir com o aumento de luz até $30 \%$ de luz, apresentando leve acréscimo em intensidades maiores de luz. A figura 7 mostra que o comportamento da MFE com aumento de luz diferiu de C. glaziuoi para C. fissilis. Enquanto na primeira espécie houve um aumento da MFE com o aumento da intensidade de luz, na segunda, não houve variação da MFE com o aumento da intensidade de luz acima de $30 \%$ de luz. B. australis mostrou significativo aumento da MFE de $2 \%\left(1,8 \pm 0,6 \mathrm{mg} \mathrm{cm}^{-2}\right)$ para $30 \%$ de luz $\left(3,3 \pm 1,7 \mathrm{mg} \mathrm{cm}^{-2}\right)$.

Densidade estomática - A figura 8 mostra o comportamento da densidade estomática com o aumento

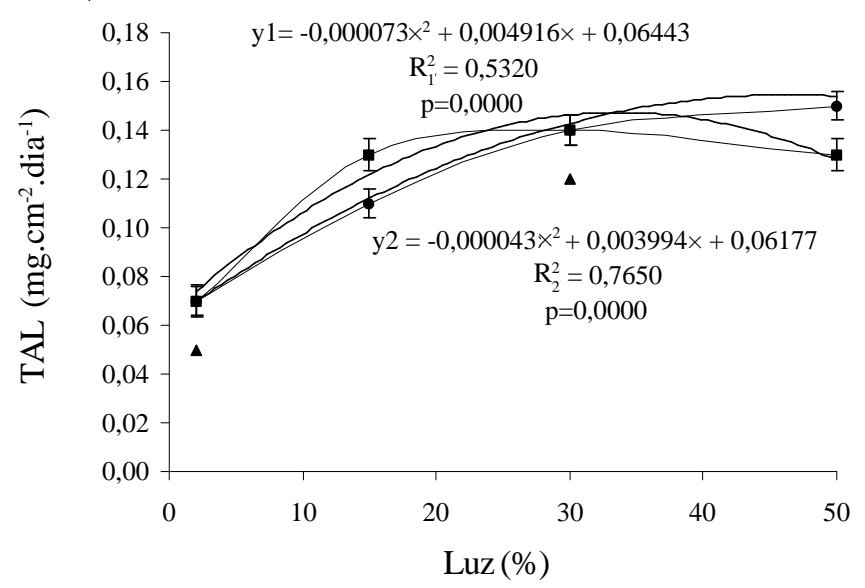

Figura 4. Taxa média de assimilação líquida (TAL) de plantas de Cecropia glazioui $(\bullet)$, Cedrela fissilis (•) e Bathysa australis (^) crescidas, respectivamente por 146, 131 e 93 dias em diferentes níveis de luz. Equações de regressão para Cedrela fissilis $\left(\mathrm{y}_{1}\right)$ e para Cecropia glazioui $\left(\mathrm{y}_{2}\right)$, coeficientes de determinação para Cedrela fissilis $\left(\mathrm{R}^{2}{ }_{1}\right)$ e para Cecropia glazioui $\left(\mathrm{R}_{2}^{2}\right)$. Modelos e coeficientes significativos em nível de $5 \%$ de probabilidade. Os índices de dispersão correspondem ao erro padrão da média.

Figure 4. Mean unit leaf rate (TAL) of Cecropia glazioui $(\bullet)$, Cedrela fissilis (-) and Bathysa australis ( $\mathbf{(})$ seedlings growing respectively during 146,131 and 93-day period under different light conditions. Regression equations for Cedrela fissilis $\left(\mathrm{y}_{1}\right)$ and for Cecropia glazioui $\left(\mathrm{y}_{2}\right)$, regression coefficients for Cedrela fissilis $\left(\mathrm{R}^{2}{ }_{1}\right)$ and for Cecropia glazioui $\left(\mathrm{R}_{2}^{2}\right)$. Regression curves and regression coefficients are significant at the 0,005 level.

da intensidade de luz. Observa-se que há uma tendência ao aumento da densidade estomática com o aumento da intensidade de luz de $2 \%$ para $30 \%$ de luz tanto em C. glazioui como em C. fissilis. Acima de $30 \%$ de luz não foi evidenciado aumento da densidade estomática com aumento da intensidade de luz. B. australis mostrou diferença significativa na densidade estomática em relação à intensidade de luz, sendo a densidade estomática menor a $2 \%$ de luz ( $92 \pm 25$ estômatos $\left.\mathrm{mm}^{-2}\right)$ que a $30 \%$ de luz ( $237 \pm 76$ estômatos $\left.\mathrm{mm}^{-2}\right)$.

Determinação do ângulo de inclinação das folhas - O ângulo medido entre a face inferior do pecíolo e o caule, em C. glazioui não apresentou diferença significativa entre plantas a $2 \%$ e $50 \%$ de luz, entretanto o ângulo medido entre a face abaxial da lâmina foliar e o pecíolo foi maior em plantas crescidas a $2 \%$ de luz $\left(120^{\circ} \pm 23\right)$ que a $50 \%$ de luz $\left(102^{\circ} \pm 14,7\right)$ fazendo com que as folhas ficassem mais perpendiculares ao solo no primeiro caso. 


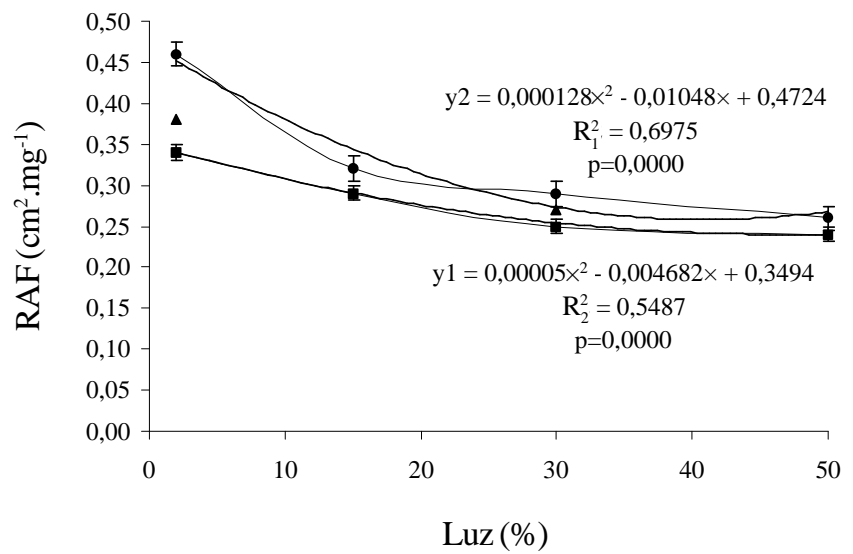

Figura 5. Razão média de área foliar (RAF) de plantas de Cecropia glazioui $(\bullet)$, Cedrela fissilis (•) e Bathysa australis (४) crescidas, respectivamente por 146, 131 e 93 dias em diferentes níveis de luz. Equações de regressão para Cedrela fissilis $\left(\mathrm{y}_{1}\right)$ e para Cecropia glazioui $\left(\mathrm{y}_{2}\right)$, coeficientes de determinação para Cedrela fissilis $\left(\mathrm{R}_{1}^{2}\right)$ e para Cecropia glazioui $\left(\mathrm{R}_{2}^{2}\right)$. Modelos e coeficientes significativos em nível de $5 \%$ de probabilidade. Os índices de dispersão correspondem ao erro padrão da média.

Figure 5. Mean leaf area ratio (RAF) of Cecropia glazioui $(\bullet)$, Cedrela fissilis (-) and Bathysa australis (४) seedlings growing respectively during 146, 131 and 93-day period under different light conditions. Regression equations for Cedrela fissilis $\left(\mathrm{y}_{1}\right)$ and for Cecropia glazioui $\left(\mathrm{y}_{2}\right)$, regression coefficients for Cedrela fissilis $\left(\mathrm{R}^{2}{ }_{1}\right)$ and for Cecropia glazioui $\left(\mathrm{R}_{2}{ }_{2}\right)$. Regression curves and regression coefficients are significant at the 0,005 level.

\section{Discussão}

Alocação de biomassa e crescimento - Quando sob baixa quantidade de luz as espécies tendem a aumentar a absorção de luz, alocando mais biomassa para as folhas que para raízes e aumentando a área foliar, o que resulta em folhas mais finas e maiores (baixa MFE), em maior RAF e em menor R/PA (Poorter 1999); quando sob alta quantidade de luz, as espécies tendem a restringir a transpiração e aumentar a capacidade fotossintética, através do aumento da MFE, resultando em folhas menores e mais grossas, menor RAF e maior razão R/PA (Lee et al. 1996). A alta razão R/PA é resultado de maior investimento de biomassa para as raízes, possibilitando maior absorção de água para suprir a demanda transpiratória em alta irradiância (Poorter 1999); alta MFE é resultado de diminuição do tamanho das folhas e de aumento na massa seca foliar, representando um aumento na quantidade e capacidade do aparato fotossintético por unidade de área (Ellsworth

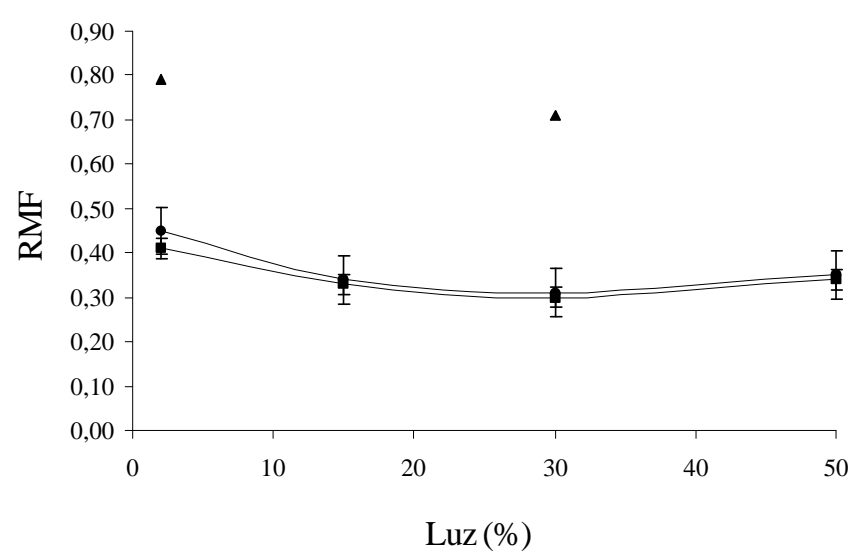

Figura 6. Razão de massa foliar (RMF) de plantas de Cecropia

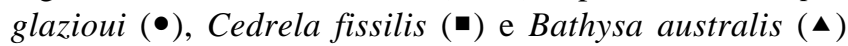
crescidas, respectivamente por 146, 131 e 93 dias em diferentes níveis de luz. Equações de regressão para Cedrela fissilis $\left(\mathrm{y}_{1}\right)$ e para Cecropia glazioui $\left(\mathrm{y}_{2}\right)$, coeficientes de determinação para Cedrela fissilis $\left(\mathrm{R}^{2}{ }_{1}\right)$ e para Cecropia glazioui $\left(\mathrm{R}_{2}{ }_{2}\right)$. Modelos e coeficientes significativos em nível de $5 \%$ de probabilidade. Os índices de dispersão correspondem ao erro padrão da média.

Figure 6. Leaf weight ratio (RMF) of Cecropia glazioui $(\bullet)$, Cedrela fissilis $(\bullet)$ and Bathysa australis $(\mathbf{\Lambda})$ seedlings growing respectively during 146, 131 and 93-day period under different light conditions. Regression equations for Cedrela fissilis $\left(\mathrm{y}_{1}\right)$ and for Cecropia glazioui $\left(\mathrm{y}_{2}\right)$, regression coefficients for Cedrela fissilis $\left(\mathrm{R}^{2}{ }_{1}\right)$ and for Cecropia glazioui $\left(\mathrm{R}_{2}{ }_{2}\right)$. Regression curves and regression coefficients are significant at the 0,005 level.

\& Reich 1992). A diminuição do tamanho das folhas reduz a camada adjacente entre atmosfera e folha, permitindo maior perda de calor por convecção para o ambiente, sendo necessária, dessa maneira, menor transpiração para resfriar a folha (Poorter 1999). Na fase de crescimento estudada, as três espécies tiveram plasticidade para alterar a RAF, a MFE, a RMF e a razão R/PA quando a intensidade de luz variou dentro de $2 \%$ para $30 \%$ ou para $50 \%$, podendo com estas alterações morfológicas, aumentar a captação de luz, quando em menor irradiância, e diminuir a transpiração, quando em maior irradiância.

A taxa de assimilação líquida (TAL) é um reflexo da capacidade fotossintética da planta em relação à área fotossintetizante (Hunt 1982). Neste estudo, as três espécies aumentaram a capacidade fotossintética com o aumento da irradiância de $2 \%$ para $30 \%$. Quando outras irradiâncias foram experimentadas, como no caso de $C$. glazioui e $C$. fissilis, a primeira foi capaz de aumentar a capacidade fotossintética com aumento na 


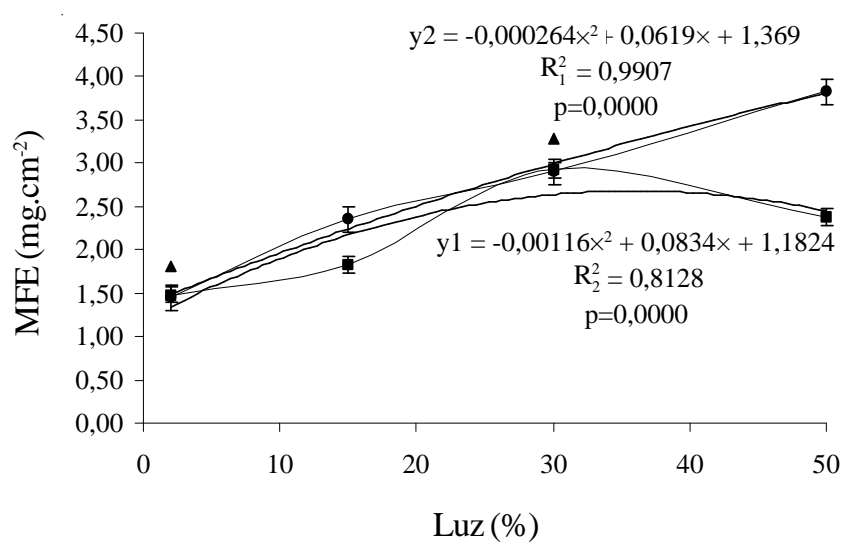

Figura 7. Massa foliar específica (MFE) de plantas de Cecropia glazioui $(\bullet)$, Cedrela fissilis ( $\bullet$ e Bathysa australis (ム) crescidas, respectivamente por 146, 131 e 93 dias em diferentes níveis de luz. Equações de regressão para Cedrela fissilis $\left(\mathrm{y}_{1}\right)$ e para Cecropia glazioui $\left(\mathrm{y}_{2}\right)$, coeficientes de determinação para Cedrela fissilis $\left(\mathrm{R}^{2}{ }_{1}\right)$ e para Cecropia glazioui $\left(\mathrm{R}_{2}{ }_{2}\right)$. Modelos e coeficientes significativos em nível de $5 \%$ de probabilidade. Os índices de dispersão correspondem ao erro padrão da média.

Figure 7. Specific leaf weight (MFE) of Cecropia glazioui $(\bullet)$, Cedrela fissilis (-) and Bathysa australis ( $\bullet$ ) seedlings growing respectively during 146, 131 and 93-day period under different light conditions. Regression equations for Cedrela fissilis $\left(\mathrm{y}_{1}\right)$ and for Cecropia glazioui $\left(\mathrm{y}_{2}\right)$, regression coefficients for Cedrela fissilis $\left(\mathrm{R}^{2}{ }_{1}\right)$ and for Cecropia glazioui $\left(\mathrm{R}_{2}{ }_{2}\right)$. Regression curves and regression coefficients are significant at the 0,005 level.

irradiância de $2 \%$ até $50 \%$ de luz, enquanto a segunda, apresentou um decréscimo da capacidade fotossintética acima de $30 \%$ de luz, o que pode indicar, para C. fissilis, ponto de saturação de luz mais baixo que o de C. glazioui. Esse resultado é coerente com a posição sucessional de C. fissilis, espécie intermediária na sucessão e com os dados de Inoue (1980), que demonstrou maior produtividade para esta espécie em menores níveis de luz.

A TCR é função da TAL e da RAF, e estes dois últimos parâmetros tendem a variar inversamente com a variação na irradiância (Osunkoya et al. 1994). Em B. australis a TCR foi mais baixa a $2 \%$ que em intensidade de luz mais elevada porque o aumento da RAF não compensou a diminuição da TAL em baixa intensidade luminosa. Os valores de TCR das outras duas espécies foram pouco afetados pelo nível de sombreamento, pois os aumentos na TAL em resposta a incrementos da intensidade luminosa foram compensados por decréscimos na RAF, especialmente

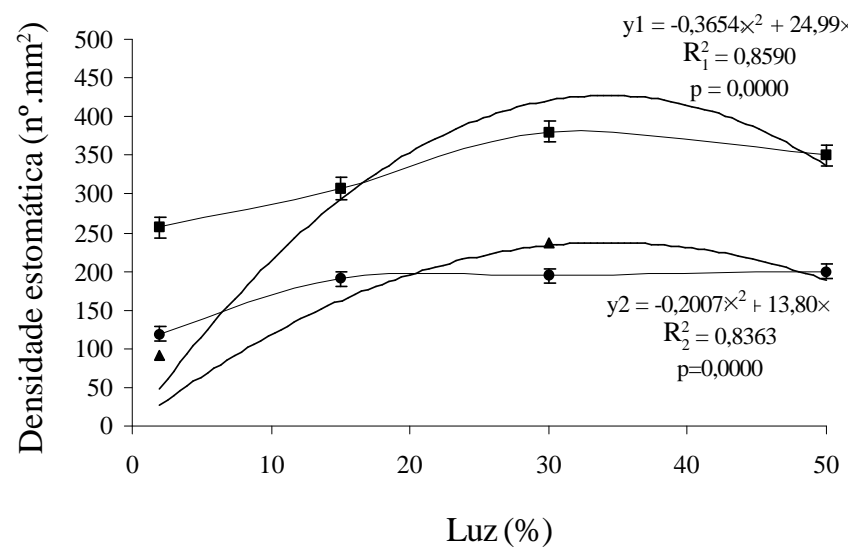

Figura 8. Densidade estomática em folhas de plantas de Cecropia glazioui $(\bullet)$, Cedrela fissilis ( $(\bullet)$ e Bathysa australis (ム) crescidas, respectivamente por 146, 131 e 93 dias em diferentes níveis de luz. Equações de regressão para Cedrela fissilis $\left(\mathrm{y}_{1}\right)$ e para Cecropia glazioui $\left(\mathrm{y}_{2}\right)$, coeficientes de determinação para Cedrela fissilis $\left(\mathrm{R}^{2}{ }_{1}\right)$ e para Cecropia glazioui $\left(\mathrm{R}_{2}{ }_{2}\right)$. Modelos e coeficientes significativos em nível de $5 \%$ de probabilidade. Os índices de dispersão correspondem ao erro padrão da média.

Figure 8. Stomatal density in leaves of Cecropia glazioui $(\bullet)$, Cedrela fissilis (-) and Bathysa australis ( $\mathbf{(})$ seedlings growing respectively during 146, 131 and 93-day period under different light conditions. Regression equations for Cedrela fissilis $\left(\mathrm{y}_{1}\right)$ and for Cecropia glazioui $\left(\mathrm{y}_{2}\right)$, regression coefficients for Cedrela fissilis $\left(\mathrm{R}^{2}\right)$ and for Cecropia glazioui $\left(\mathrm{R}_{2}{ }_{2}\right)$. Regression curves and regression coefficients are significant at the 0,005 level.

em C. fissilis. Os dados de TCR encontrados para C. glaziuoi são surpreendentes, já que é geralmente esperado que espécies pioneiras aumentem a TCR com o aumento de irradiância (Poorter 1999), em virtude de um aumento da TAL que suplante a diminuição da RAF à medida que a irradiância aumente. Uma das possíveis explicações para a falta de variação na TCR pode residir na limitação de nutrientes para o crescimento das plantas em maior intensidade de luz, dada, talvez, pelo tamanho da embalagem onde as plantas foram cultivadas. Esta condição poderia limitar o crescimento de folhas e a fotossíntese, o que levaria a baixos valores de TCR em alta irradiância. Entretanto, os valores de TCR encontrados para $C$. glazioui, neste trabalho, estão dentro do rol de valores para TCR encontrados para plântulas de espécies arbóreas de florestas tropicais. Em estudo de Souza (1996) com 15 espécies da Floresta Tropical Atlântica, de idades similares às deste trabalho, mostrou para a maioria das espécies de início de sucessão, sob cerca de $85 \%$ de irradiância, TCR entre 
0,045 e $0,035 \mathrm{mg} \mathrm{mg}^{-1} \mathrm{~d}^{-1}$, tendo C. glazioui apresentado TCR equivalente a $0,035 \mathrm{mg} \mathrm{mg}^{-1} \mathrm{~d}^{-1}$, valor semelhante ao encontrado para esta espécie neste trabalho. Poorter (1999), trabalhando com plântulas até 5 meses de idade, de espécies da Floresta Tropical Amazônica, encontrou para Cecropia ficifolia, espécie pioneira, TCR equivalente a $0,025 \mathrm{mg} \mathrm{mg}^{-1} \mathrm{~d}^{-1}$ a $25 \%$ da luz solar plena e equivalente a $0,022 \mathrm{mg} \mathrm{mg}^{-1} \mathrm{~d}^{-1}$ a pleno sol.

A intensidade de $2 \%$ da luz solar é comparável à irradiância encontrada sob dossel fechado da floresta (Lee et al. 1996) e as três espécies estudadas mostraram valor significativo de crescimento nesta baixa irradiância, a despeito de duas delas, C. glazioui e C. fissilis, serem encontradas em estádios iniciais de sucessão. $\mathrm{O}$ crescimento das plântulas nesta baixa irradiância deu-se porque as três espécies foram capazes de manter, em baixa intensidade de luz, TAL positiva. Esse comportamento de tolerância ao sombreamento tem sido detectado na maioria das arbóreas de florestas tropicais, enquanto que a intolerância à sombra, no sentido atribuído por Whitmore (1990), que é a germinação, sobrevivência e crescimento de espécies somente em clareira, tem sido detectada em poucas espécies arbóreas florestais (Poorter 1999).

Altura do caule e ângulo de inclinação das folhas Plantas crescendo sob baixa irradiância podem apresentar caules mais altos que aquelas sob alta irradiância, a despeito das primeiras apresentarem menor biomassa (Felfili et al. 1999). Essa resposta à baixa irradiância é consequiência da diminuição da quantidade de luz azul, percebida pelo pigmento criptocromo, ou do aumento da proporção de radiação vermelho-extremo em relação à radiação vermelha, percebido pelo pigmento fitocromo (Ballaré et al. 1997). Embora, neste estudo, a razão vermelho/vermelho-extremo (V/VE) tenha sido similar a pleno sol $(\mathrm{V} / \mathrm{VE}=1,10)$ e sob as telas sombrite de diferentes cortes de luz (V/VE $=1,09$ a $50 \%, 30 \%$ e $15 \%$ da luz solar e 0,93 a $2 \%$ da luz solar), essa razão pode ter sido alterada ao nível das plantas em cada tratamento, uma vez que diferenças na proximidade das plantas, causadas pela maior ou menor área foliar em cada nível de luz, podem causar alteração na reflexão de vermelho e vermelho-extremo, alterando a razão V/VE entre os diferentes níveis de luz (Ballaré et al. 1997). As três espécies mostraram diferentes respostas em altura do caule com o aumento de irradiância. Em C. glazioui houve diminuição do comprimento do caule com o aumento da irradiância, em C. fissilis, entretanto, a relação foi inversa até $15 \%$ de luz, com a espécie apresentando aumento de altura de caule na medida em que aumentou a irradiância. $B$. australis não alterou a altura do caule com a variação de irradiância. A resposta de aumento de altura do caule com a diminuição de luz, exibido por $C$. glazioui, tem sido associada a espécies arbóreas mais exigentes por luz, que com esse tipo de comportamento podem rapidamente superar em altura as plantas herbáceas que as sombreiam (Poorter 1999).

A sensibilidade das espécies em reorientar as folhas com a variação da irradiância diferiu. C. fissilis e B. australis não mostraram reorientação das folhas com a alteração da irradiância, enquanto $C$. glazioui tornou as folhas mais perpendiculares ao solo em irradiâncias mais altas. Esta torção das folhas de acordo com a irradiância também foi observada em Cecropia obtusifolia Bertol. (Ackerly \& Bazzaz 1995) e em Stokesia laevis Hill (Callan \& Kennedy 1995), sendo relacionada à diminuição da transpiração, já que esse comportamento evita que os raios solares atinjam perpendicularmente a folha (Callan \& Kennedy 1995). Densidade dos estômatos - A densidade estomática costuma aumentar em plantas submetidas a altas irradiâncias (Lee et al. 1997), podendo resultar tanto em um aumento na absorção de $\mathrm{CO}_{2}$ (Abrans et al. 1992), quanto em uma diminuição na transpiração, este último fato devido à sobreposição da área de difusão do vapor d'água em virtude da maior proximidade dos estômatos (Larcher 1995). As três espécies estudadas foram capazes de aumentar a densidade estomática com aumento da irradiância, pelo menos até $30 \%$ de luz. Plasticidade de variação dos parâmetros analisados A plasticidade das espécies em variar determinado parâmetro em função da irradiância foi dependente do gradiente de irradiância aplicado. Percebe-se, através das curvas que representam o comportamento de cada parâmetro em função dessa variável, que, para todos os parâmetros avaliados, o grau de plasticidade foi maior (houve maior linearidade entre o aumento de irradiância e a resposta do parâmetro avaliado) nas irradiâncias mais baixas. Maior plasticidade fenotípica das espécies em gradiente mais baixo de irradiância é relatada também para outras espécies, como no estudo de Veenendaal et al. (1996), envolvendo 15 espécies arbóreas africanas. Esse dado foi também observado em Euterpe edulis Mart., espécie arbórea da Floresta Atlântica, em estudo feito por Nakazono et al. (2000). Onde houve maior linearidade entre variação do parâmetro e variação da irradiância, percebe-se que C. glazioui mostra para os parâmetros analisados, à exceção da densidade estomática, maior plasticidade (maior inclinação da reta) ou plasticidade semelhante (inclinação semelhante entre as duas retas) à de 
C. fissilis para aclimatar-se à maior irradiância. A densidade estomática, no entanto, mostrou-se ser mais plástica em C. fissilis que em C. glazioui.

Respostas das espécies à variação na irradiância versus ambiente de ocorrência - As respostas apresentadas por plântulas de $C$. glazioui à alta irradiância são coerentes com sua ocorrência em estádio inicial de sucessão, já que a espécie apresentou características particulares para aproveitar melhor a alta luminosidade, como a capacidade em aumentar a TAL em mais luz, e mecanismos para evitar a transpiração excessiva em ambientes de alta irradiação, como rotação da folha, presença de camada densa de pêlos nas folhas e expressiva diminuição da RAF. O alongamento do caule em baixa irradiância, que permite acesso mais rápido à luz, quando ocorre sombreamento por outras espécies, também é uma característica de espécies que demandam alta irradiância para seu crescimento (Poorter 1999). C. fissilis, segundo os dados encontrados neste trabalho, também apresentou plasticidade para aumentar a capacidade fotossintética e diminuir a transpiração, quando em maior irradiância, características condizentes com seus locais de ocorrência, que são clareiras, bordas de mata e matas secundárias, ambientes onde a intensidade de luz ainda é considerável. Os dados encontrados para as respostas de B. australis em relação à variação de luz não são contraditórios com sua ocorrência no subosque: exibe TCR positiva em baixa irradiância e é capaz de aproveitar um aumento na irradiância devido ao aparecimento de clareiras, aumento, este, necessário ao recrutamento de plântulas de muitas espécies de estádio sucessional tardio (Whitmore 1990).

Agradecimentos - Os autores agradecem à Capes pelo auxílio financeiro para o desenvolvimento do trabalho, ao Prof. Ademir Reis pela análise crítica do manuscrito, ao Prof. Maurício Sedrez dos Reis pela orientação na análise estatística dos dados e ao Instituto de Botânica de São Paulo pelo empréstimo do quantômetro para medidas de V/VE. S.R. Duz agradece à Capes e A. Siminski ao CNPq pelas bolsas de estudo concedidas durante a realização deste trabalho.

\section{Referências bibliográficas}

ABRANS, M.C., KLOEPPEL, B.D. \& KUBISKE, M.E. 1992. Ecophysiological and morphological responses to shade and drought in two contrasting ecotypes of Prunus serotina. Tree Physiology 10:343-355.

ACKERLY, D.D. \& BAZZAZ, F.A. 1995. Seedling crown orientation and interception of diffuse radiation in tropical forest gaps. Ecology 76:1134-1146.
BALLARÉ, C.L., SCOPEL, A.L. \& SÁNCHEZ, R.A. 1997. Foraging for light: photosensory ecology and agricultural implications. Plant, Cell and Environment 20: 820-825.

CALLAN, E.J. \& KENNEDY, C.W. 1995. Intercropping stokes aster: Effect of shade on photosynthesis and plant morphology. Crop Science 35:1110-1115.

CENTENO, A.J. 1982. Curso de estatística aplicada à Biologia. Editora Universidade Federal de Goiás, Goiânia.

DURIGAN, G., FIGLOLIA, M.B., KAWABATA, M., GARRIDO, M.A.O., BAITELLO, J.B. 1997. Sementes e mudas de árvores tropicais. Páginas e Letras Editoras Gráficas, São Paulo.

EASTMAN, J.R. 1997. Idrisi for Windows version 2.0. Clark University, Worcester.

ELLSWORTH, D.S. \& REICH, P.B. 1992. Leaf mass per area, nitrogen content and photosynthetic carbon gain in Acer saccharum seedlings in contrasting forest light environments. Functional Ecology 6:423-435.

FELFILI, J.M., HILGBERT, L.F., FRANCO, A.C., SOUSASILVA, J.C., REZENDE, A.L. \& NOGUEIRA, M.V.P. 1999. Comportamento de plântulas de Sclerolobium paniculatum Vog. var. rubiginosum (Tul.) Benth. sob diferentes níveis de sombreamento, em viveiro. Revista Brasileira de Botânica 22:297-301.

HUNT, R. 1982. Plant growth curves. The functional approach to plant growth analysis. Edward Arnold, London.

INOUE, M.T. 1980. Photosynthesis and transpiration in Cedrela fissilis Vell. seedlings in relation to light intensity and temperature. Turrialba 30:280-283.

ISHIDA, A., TOMA, T. \& MARJENAH. 1999. Limitation of leaf carbon gain by stomatal and photochemical processes in the canopy of Macaranga conifera, a tropical pioneer. Tree Physiology 19:467-473.

JOHANSEN, D.A. 1940. Plant microtechnique. McGraw-Hill, New York.

KLEIN, R.M. 1984. Meliáceas. Flora Ilustrada Catarinense. MELI. Herbário Barbosa Rodrigues, Itajaí.

KITAJIMA, K. 1996. Ecophysiology of tropical tree seedlings. In Tropical forest plant ecophysiology (S.S. Mulkey, R.L Chazdon \& A.P. Smith, eds.). Chapman \& Hall, New York, p.559-595.

LARCHER, W. 1995. Physiological Plant Ecology. SpringerVerlag, Berlin.

LEE, D.W., BASKARAN, K., MANSOR, M., MOHAMAD, H. \& YAP, S.K. 1996. Irradiance and spectral quality affect Asian tropical rain forest tree seedling development. Ecology 77:568-580.

LEE, D.W., OBERBAUER, S.F., BASKARAN, K., MANSOR, M., MOHAMAD, H. \& YAP, S.K. 1997. Effects of irradiance and spectral quality on seedling development of two Southeast Asian Hopea species. Oecologia 110:1-9. 
LEE, D.W., OBERBAUER, F., JOHNSON, P., BASKARAN, K., MANSOR, M., MOHAMAD, H. \& YAP, S.K. 2000. Effects of irradiance and spectral quality on leaf structure and function in seedlings of two Southeast Asian Hopea (Dipterocarpaceae) species. American Journal of Botany 87:447-455.

LORENZI, H. 1998. Árvores Brasileiras, manual de identificação e cultivo de arbóreas nativas do Brasil. v.1, 2 ed. Plantarum. Nova Odessa.

MATTHES, L.A.F. \& MARTINS, F.R. 1996. Conceitos em sucessão ecológica. Revista Brasileira de Horticultura Ornamental 2:19-32.

NAKAZONO, E.M., COSTA, M.C., FUTATSUGI, K. \& PAULILO, M.T.S. 2001. Crescimento inicial de Euterpe edulis Mart. em diferentes regimes de luz. Revista Brasileira de Botânica 24:173-179.

OSUNKOYA, O.O., ASH, J.E., HOPKINS, M.S. \& GRAHAM, A.W. 1994. Influence of seed size and seedling ecological attributes on shade-tolerance in northern Queensland. Journal of Ecology 82:149-163.

PETIT, C., THOMPSON, J.D. \& BRETAGNOLLE, F. 1996. Phenotipic plasticity in relation to ploydy level and corn production in the perennial grass Arrhenatherum elatius. Canadian Journal of Botany 74:1964-1963.

POORTER, L. 1999. Growth responses of 15 rain-forest tree species to a light gradient : the relative importance of morphological and physiological traits. Functional Ecology 13:396-410.
SOUZA, R.P. 1996. Germinação, crescimento, atividade fotossintética e translocação de compostos de carbono em espécies arbóreas tropicais: estudo comparativo e influência de sombreamento natural. Tese de doutorado, Universidade Estadual de Campinas, Campinas.

STRAUSS-DEBENEDETTI, S. \& BAZZAZ, F. 1996. Photosynthetic characteristics of tropical trees along sucessional gradients. In Tropical forest plant ecophysiology (S.S. Mulkey, R.L. Chazdon \& A.P. Smith, eds.). Chapman \& Hall, New York, p.162-186

TABARELLI, M., VILANI, J.P. \& MANTOVANI, W. 1993. Aspectos da sucessão secundária em trecho da Floresta Atlântica no Parque Estadual da Serra do Mar, SP. Revista do Instituto Florestal 5:99-102.

VÁLIO, I.F.M. \& SCARPA, F.M. 2001. Germination of seeds of tropical pioneer species under controlled and natural conditions. Revista Brasileira de Botânica 24:79-84.

VEENENDAAL, E.M., SWAINE, M.D., LECHA, R.T., FALSCH, M.F., ABEBRESE, I.K. \& OWUSU-AFRIYIE, K. 1996. Responses of West African forest tree seedlings to irradiance and soil fertility. Functional Ecology 10:501-511.

WHITMORE, T.C. 1990. An introduction to tropical rain forest. Clarendon Press, Oxford. 